\title{
Enfermedad coronaria en pacientes candidatos a reemplazo percutáneo de válvula aórtica: qué debemos y qué no debemos hacer, desde la evidencia clínica al sentido común
}

\author{
Coronary disease in patients eligible for percutaneous aortic valve \\ replacement: what we should and should not do, from clinical evidence \\ to common sense
}

Revista Argentina de Cardioangiología Intervencionista 2017;8(4):175-177

Desde hace mucho tiempo la enfermedad coronaria es un acompañamiento frecuente de la estenosis valvular aórtica: entre 40 a $75 \%$ de los pacientes candidatos a reemplazo percutáneo valvular aórtico (TAVR) presentan enfermedad coronaria asociada $(\mathrm{ECA})^{1-3}$.

Sabemos que la cirugía combinada en los casos de reemplazo valvular aórtico quirúrgico incrementa en forma significativa la morbimortalidad, al menos en algunas series como las de nuestro país, donde la mortalidad sube del $8,9 \%$ cuando es reemplazo aislado al $13.4 \%$ cuando se trata de cirugías combinadas ${ }^{4}$ (Tabla 1). La introducción del TAVR hace 15 años introdujo un nuevo dilema: qué hacer con aquellos pacientes que al momento del TAVR presentan además ECA, enfermedad que no está muchas veces bien definida angiográficamente. De hecho, la severidad se define como lesión obstructiva igual o mayor al 50\% y los trabajos no definen tampoco qué tipo de lesiones son las seleccionadas para intervención coronaria percutánea (PCI) previo al TAVR. 5 .-8

Si revisamos la literatura, encontraremos que los datos son dispares y para todos los gustos, como la vieja teoría del "vaso medio lleno o medio vacío".

Existen trabajos a favor de tratar con angioplastia previamente al TAVR a aquellos que presentan ECA, así como otros estudios que no recomiendan la angioplastia previo al TAVR en tales pacientes, donde inclusive en largas series el grado de revascularización coronaria residual (residual completness of revascularization) no afecta la sobrevida a largo plazo ${ }^{1}$. Sin embargo, no todos estos trabajos mencionados describen el grado de obstrucción de la enfermedad coronaria asociada a la valvulopatía aórtica, elemento que es esencial al momento de tomar una actitud terapéutica ${ }^{1,8}$.

Me parece que acá debemos recurrir a lo que aprendimos en nuestra residencia de Cardiología Clínica: que el más común de los sentidos que es el "sentido común".

TABLA 1. Realidad de la cirugía cardíaca en la República Argentina. Regisro CONAREC XVI.

\begin{tabular}{|l|c|c|c|c|c|}
\hline Complicación & $\begin{array}{c}\text { Todos } \\
\mathbf{n}=\mathbf{2 5 5 3} \\
\mathbf{n}(\%)\end{array}$ & $\begin{array}{c}\text { CRM } \\
\mathbf{n}=1465 \\
\mathbf{n}(\%)\end{array}$ & $\begin{array}{c}\text { RVA } \\
\mathbf{n}=359 \\
\mathbf{n}(\%)\end{array}$ & $\begin{array}{c}\text { CVM } \\
\mathbf{n}=169 \\
\mathbf{n}(\%)\end{array}$ & $\begin{array}{c}\text { Combinadas } \\
\mathbf{n}=312 \\
\mathbf{n}(\%)\end{array}$ \\
\hline IAM posoperatorio & $123(4,82)$ & $87(5,94)$ & $8(2,23)$ & $4(2,37)$ & $17(5,45)$ \\
\hline SBVM & $590(23,1)$ & $241(16,45)$ & $63(17,55)$ & $58(34,32)$ & $129(41,3)$ \\
\hline Insuficiencia renal & $339(13,3)$ & $119(8,81)$ & $43(11,98)$ & $26(15,38)$ & $73(23,4)$ \\
\hline ACV & $28(1,1)$ & $9(0,61)$ & $8(2,23)$ & $5(2,96)$ & $8(2,56)$ \\
\hline Mediastinitis & $48(1,88)$ & $27(1,84)$ & $11(3,06)$ & $1(0,59)$ & $5(1,6)$ \\
\hline Sepsis & $114(4,5)$ & $44(3)$ & $21(5,85)$ & $6(3,55)$ & $24(7,7)$ \\
\hline Distrés respiratorio & $93(3,64)$ & $44(3)$ & $16(4,46)$ & $7(4,14)$ & $14(4,5)$ \\
\hline Reoperación & $147(5,76)$ & $62(4,23)$ & $21(5,85)$ & $12(7,1)$ & $34(9,77)$ \\
\hline Complicaciones mayores (global) & $809(31,7)$ & $366(24,98)$ & $105(29,2)$ & $67(39,64)$ & $154(49,36)$ \\
\hline Mortalidad & $196(7,68)$ & $63(4,3)$ & $32(8,91)$ & $18(10,65)$ & $42(\mathbf{3}, 4)$ \\
\hline
\end{tabular}

Modificado de: Lowenstein et al. Registro CONAREC XVI. Rev Argent Cardiol. 2010;78:228-37.

CRM: cirugía de revascularización miocárdica. RVA: remplazo valvular aórtico. CVM: cirugía valvular mitral. IAM: infarto agudo de miocardio. SBVM: síndrome de baio volumen minuto. ACV: accidente cerebrovascular. 


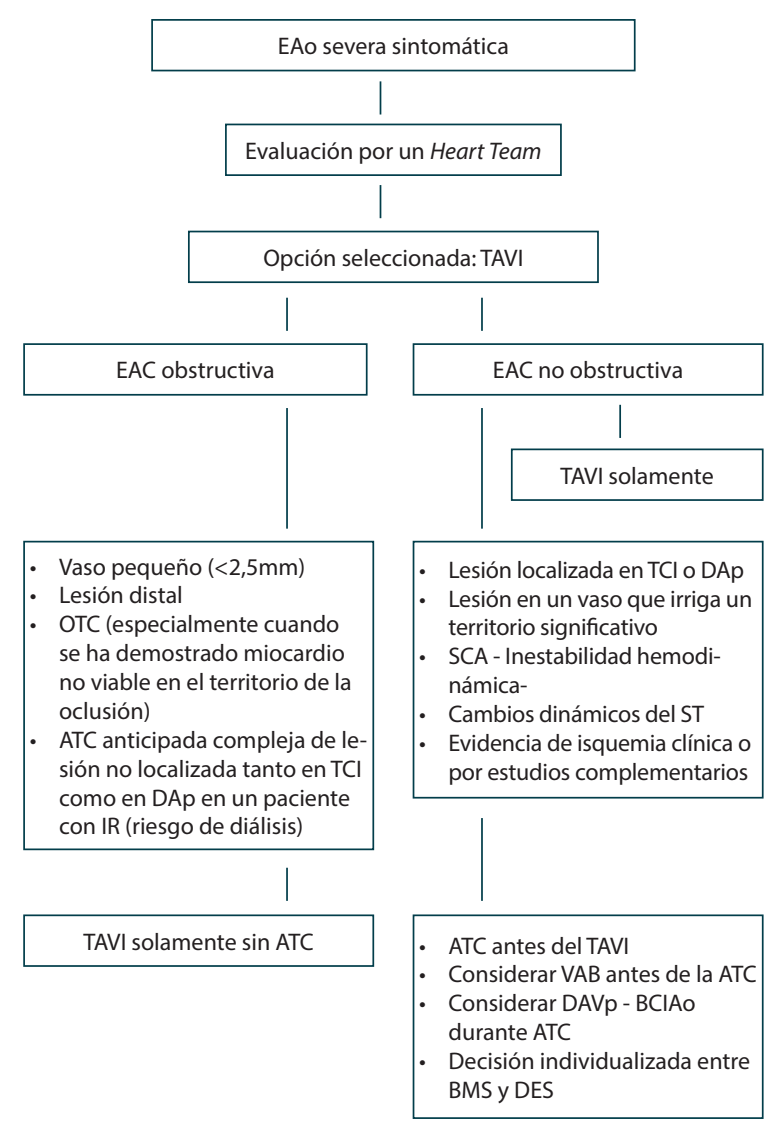

Figura 1. Algoritmo de tratamiento en pacientes candidatos a TAVR propuesto por la Universidad de Columbia
¿Cuál es el motivo de consulta del paciente, cuál es el síntoma predominante? ¿Es insuficiencia cardíaca, angor, síncope o una mezcla de todos?

Si el paciente llega a nuestra consulta por enfermedad estenótica de la válvula aórtica, es entonces este hallazgo el que deberemos priorizar. Si por el contrario la estenosis aórtica es un epifenómeno descubierto en un paciente con enfermedad coronaria severa conocida, estamos ante otro paciente.

Cuando vamos a tratar estenosis de arterias coronarias, debemos recordar lo que aprendimos de los diversos estudios del FAME 9 usando test funcionales intracoronarios para evaluar lesiones isquémicas y también debemos reconocer los estudios locales y multicéntricos ERACI y WALTZ ${ }^{10}$, donde lesiones intermedias no tratadas con angioplastia tuvieron a 3 años y 11 meses, respectivamente, solo 3,8\% y $1 \%$ de necesidad de implante de stent, lo que sugiere que la mayoría de las lesiones intermedias no necesita ser tratada. Es muy interesante observar el algoritmo de tratamiento en pacientes candidatos a TAVR propuesto por la Universidad de Columbia ${ }^{11}$ que se puede observar en la Figura 1, donde claramente identifican a las lesiones coronarias localizadas en vasos $<2,5 \mathrm{~mm}$ de diámetro, oclusiones totales crónicas irrigando áreas miocárdicas no viables, presencia de severas enfermedades concomitantes como insuficiencia renal severa etc. como solo candidatos a TAVR sin realizar PCI previa. El otro hecho a tener en cuenta en estos pacientes añosos con severas comorbilidades y alto riesgo de sangrado es el tipo de stent que vamos a utilizar dada la necesidad de muchos de ellos de la triple antiagregación plaquetaria con el consiguiente riesgo de sangrado. Como ejemplo, el peor escenario es un paciente que consultó por estenosis aórtica severa y como hallazgo se detectaron lesiones coronarias intermedias, que erróneamente fueron tratadas con implante de stents; y dada la necesidad de anticoagulación, este paciente presentó como complicación hemorragia intestinal severa por angiodisplasia colónica ${ }^{12}$, algo que es frecuente en pacientes añosos con estenosis aórtica. Como consecuencia, al "final del día" este paciente con estenosis aórtica crítica está siendo tratado por enfermedades concomitantes provocadas por una incorrecta evaluación e indicación médica, dejando sin tratar la patología aórtica que lo llevó a la consulta: como dijimos antes, el más común de los sentidos, "el sentido común", debe también estar presente a la hora de las indicaciones terapéuticas.

Por lo anteriormente descripto, es de real importancia identificar cuál fue el motivo de la consulta clínica además de analizar otras variables como edad, actividad física, perspectiva de vida, etc. antes de tomar una conducta clínica.

Además debemos enfatizar en que el implante TAVR no contraindica de ninguna manera una angioplastia posterior. El ejemplo de esto se muestra en la Figura 2, donde un paciente con lesión menos que intermedia en la arteria descendente anterior no considerada para implante de stent en nuestro servicio y a quien se le realizó implante exitoso de TAVR, presentó una significativa progresión de la enfermedad tres meses después de la TAVR que evolucionó a un síndrome coronario agudo, que necesitó un implante de stent que no fue motivo de dificultad por la válvula aórtica antes implantada.

En síntesis, lo primero que debemos enseñar a nuestros jóvenes cardiólogos intervencionistas es el criterio clínico. Primero, es muy importante identificar cuáles son los síntomas que traen a nuestros candidatos para TAVR, así como todas las variables clínicas asociadas, edad, actividad física, perspectiva de vida, etc. Luego de ello, es también importante reconocer que la mayoría de las lesiones crónicas "intermedias", que serían el caso de aquellos pacientes candidatos primariamente a TAVR, en su gran mayoría no van a necesitar más que estatinas, el ejemplo de la Figura 2 es una excepción a tener en cuenta que vale solo como ejemplo aislado. Recordemos nuevamente que estos eventos sobre lesiones crónicas no isquémicas tuvieron en el estudio ERACI IV ${ }^{10}$ una recurrencia isquémica a 3 años del $3,8 \%$. 


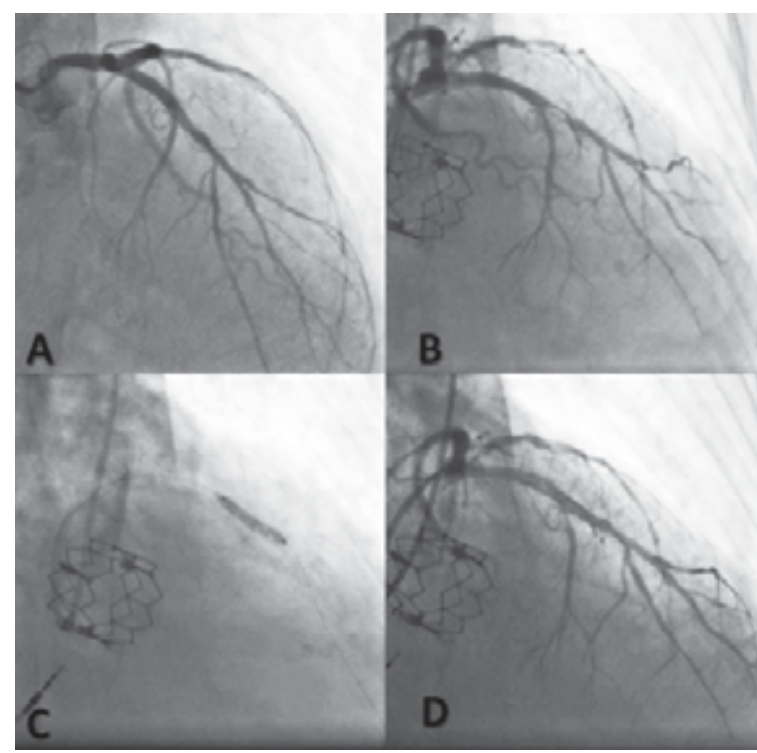

Figura 2. Progresión de enfermedad coronaria en paciente con TAVI
Finalmente, el uso de métodos como el flujo de reserva miocárdica para evaluar las lesiones podría usarse fácilmente, aunque incrementaría los costos, y en estos pacientes con estenosis aórtica pueden infravalorar el grado de isquemia miocárdica.

Resumiendo, y para recomendación especialmente a nuestros jóvenes fellows de la Carrera, ante la presencia de una estenosis valvular severa candidato a TAVR y que presenta ECA:

La real incidencia de ECA definida como lesión obstructiva $>70 \%$ en pacientes candidatos a TAVR no la conocemos.

Una inadecuada lectura de la cinecoronariografía previa al TAVR puede trasladarse en una conducta médica incorrecta y muchas veces la misma ser dañosa para el paciente.

Se deberían utilizar scores angiográficos anatómicos para determinar el grado de riesgo concomitante de la ECA antes de cualquier actitud terapéutica, teniendo en cuenta el grado de severidad de la estenosis coronaria (>70\%), la extensión del área miocárdica afectada, las comorbilidades no cardíacas asociadas frecuentemente en estos pacientes muy añosos, así como el eventual uso de stents ya sea convencionales o liberadores de fármacos pero que tengan suficiente evidencia clínica de seguridad en pacientes con alto riesgo de sangrado. La angioplastia coronaria postimplante de TAVR se puede realizar con éxito y aparentemente sin mayores dificultades, dependiendo del tipo de válvula, aunque la experiencia es limitada al momento presente.

Hay en curso tres estudios aleatorizados que especificamente están buscando respuesta a estos interrogantes ( $A C$ TIVATION, PARTNER 2 A y SURTAVI trials) y que quizás nos brinden una mayor base científica sobre cuál es en definitiva la mejor actitud ante estos pacientes.

Alfredo E. Rodríguez MD, PhD, FACC, FSCAI

Editor en Jefe de la Revista Argentina de Cardioangiología Intervencionista (RACI)

\section{BIBLIOGRAFÍA}

1. Van Mieghem $N$, van der Boon $R$, Fagiri E, et al. Complete revascularization /s not a prerequisite for success in current transcatheter aortic valve implantation practice. J Am Coll Cardiol Intv 2013;6:867-75.

2. Goel S, Ige M, Tuzcu EM, et al. Severe aortic stenosis and coronary artery disease-implicationsformanagement in the transcatheteraortic valvereplacement era: a comprehensive review. J Am Coll Cardiol 2013;62:1-10.

3. Wenaweser P, Pilgrim T, Guerios E, et al. Impact of coronary artery disease and percutaneous coronary intervention on outcomes in patients with severe aortic stenosis undergoing transcatheter aortic valveimplantation. Eurolntervention 2011;7:541-8.

4. Lowenstein D, Guardiani F, Pieroni P, et al. Realidad de la Cirugía Cardíaca en la Argentina: Registro CONAREC XVI. Rev Argent de Cardiol 2010;78:228-37.

5. Abramowitz Y, Banai S, Katz G, et al. Comparison of early and late outcomes of TAVI alone compared to TAVI plus PCl in aortic stenosis patients with and without coronary artery disease. Catheter Cardiovasc Interv 2014;83:649-54.

6. HuczekZ, Zbroński K, Grodecki K, et al. Concomitant coronary artery disease and its management in patients referred to transcatheter aortic valveimplantation: insights from the POL-TAVI Registry. Catheter Cardiovasc Interv 2017 Aug 26. doi: $10.1002 /$ ccd.27251.

7. Dewey T, Brown D, Herbert M, et al. Effect of concomitant coronary artery di- sease on procedural and late outcomes of transcatheter aortic valve implantation. Ann Thorac Surg 2010;89:758-67.

8. Paradisa JM, Labbéa B, Rodés-Cabaua J. Coronary artery disease and transcatheteraortic valvereplacement: currenttreatmentparadigms. Coronary Artery Disease 2015;26:272-8.

9. Stefanini G, Stortecky S, Wenaweser P, Windecker S, Coronary artery disease in patients undergoing TAVI: why, what, when and how to treat. Eurolntervention 2014;10:U69-U75.

10. Kobayashi Y, Nam CW, Tonino PA, FAME Study Investigators, et al. The prognostic value of residual coronary stenoses after functionally complete revascularization. J Am Coll Cardiol 2016;67:1701-11.

11. Rodriguez AE, Santaera O, Larribau M, et al. Second versus first drug eluting stents in complex lesions subsets: 3 years follow up of ERACIIV Study. Minerva Cardioangiol 2017;65(1):81-90.

12. Paradis JM, Fried J, Nazif T, et al. Aortic stenosis and coronary artery disease: What do we know? What don't we know? A comprehensive review of the literature with proposed treatment algorithms. European Heart Journal doi:10.1093/eurheartj/ehu247

13. Cappell MS, Lebwohl O. Cessation of recurrent bleeding from gastrointestinal angiodysplasias after aortic valve replacement. Ann Intern Med 1986;105(1):54-7. 